\title{
SUPER-RESOLUTION IN MEDICAL IMAGING : AN ILLUSTRATIVE APPROACH THROUGH ULTRASOUND
}

\author{
D. Kouamé
}

\author{
University of Toulouse, IRIT UMR CNRS 5505, \\ Toulouse France
}

\author{
M. Ploquin \\ University of Tours, UMR INSERM U930, \\ Tours france
}

\begin{abstract}
The requirements for better resolution in all medical imaging modalities currently represent a very important and open challenge. Accurate measurement and visualization of structure in living tissues is intrinsically limited by the imaging system features. Imaging beyond these limits in medical imaging is referred to as super-resolution. We provide first a brief overview of super-resolution imaging and present a technique for achieving super-resolution in medical imaging based on analysis of the imaging system point spread function, and illustrate the methodology in the case of ultrasound imaging. The technique proposed uses parametric modeling to estimate B-mode images. The technique behavior is illustrated using phantom and in vivo images.
\end{abstract}

Index Terms - AR model, Medical Imaging, Multidimensional, Super-resolution, Ultrasound.

\section{INTRODUCTION}

The resolution of an imaging system is defined by its ability to separate two close point sources. The term super-resolution (SR) may have different meanings depending on the application domain or point of view. For instance, as stated by Baker and Kanade [1], almost all the algorithms dealing with SR are based on the constraints that an SR image should generate low resolution input images when appropriately warped and down-sampled to model the image formation process. Thus (conversely) SR is the process of combining multiple low resolution images to form a higher resolution image. This analysis is less valid in medical imaging. Medical images are always the result of interactions between a tissue and a physical phenomenon such as a wave (in Ultrasound, MRI) or an ionizing particle (in X-ray/CT, PET or SPECT). Due to high diagnostic potential and biological models, imaging biological micro-structures of sub-micron size has been a important goal in recent years for many imaging modalities. In noninvasive medical imaging, the physical phenomenon crosses intermediate tissues (such as bone, blood, skin, ...) before the expected interaction occurs. Loss of resolution in medical images results mainly from two limitations which are respectively intrinsic and extrinsic. Intrinsic resolution limitation originates from a physical interaction between the tissue and the physical phenomenon (wave or elementary particle). This resolution is limited both by the characteristics of this interaction, such as attenuation, scattering, etc, and by the characteristics of the sensors such as wavelength, its nature and arrangement (spatial distance between detectors). Extrinsic resolution limitation results from perturbation of the image recordings. Such resolution is limited by the non-spontaneous and spontaneous rhythms of the tissue or the patient such as movements, heart or breathing rate. Hence, general purpose super-resolution techniques are limited to extrinsic resolution enhancement. These techniques combine sets of low resolution images to obtain images with higher resolution (see e.g., [1], [2], [3] and relevant references therein, and also other papers in this special session).

Much attention has been paid to devices for the enhancement of intrinsic resolution, and many studies have been performed on the optimization of the imaging devices or sensors or are under consideration (see e.g. [4], [5], [6], [7], [8], [9], [10]).The problem of extrinsic resolution enhancement by post-processing of images rather than device-engineering challenge has recently become the focus of much interest. Most studies deal with a deconvolution-based approach (see e.g. [11]). The remainder of this paper will consider intrinsic resolution enhancement using post-processing techniques. To get better insight, we consider the case of ultrasound imaging. The objective is overcoming the physical limitations of the imaging system. One important criterion which facilitates the understanding of super-resolution is the Rayleigh diffraction limit [12]. Roughly speaking this limitation for ultrasound imaging may be considered as being $\lambda / 2$ or, since in medical imaging it is important distinguish lateral resolution $r_{l}$ from axial resolution $r_{a}$, these limit resolutions may be expressed by [8]: $r_{l}=\lambda f_{\text {number }}=\lambda \frac{L}{D} ; \quad r_{a}=\frac{1}{2} \frac{c}{B_{w}}$ where $\lambda$ is the average ultrasound wavelength, $c$ is sound velocity, $f_{\text {number }}=L / D$, and $D$ and $L$ are diameter and focal length of the transducer (sensor), respectively; $B_{w}$ is the bandwidth of the transducer. The aim of SR imaging is to provide images with resolution above these limitations. $r_{l}$ and $r_{a}$ are accessible through the point spread function (PSF) of the imaging system. Various studies have described the PSF-based resolu- 
tion properties of super-resolution [13], [14], [15], [16]. Using a resolution estimation model, we suggest here a method to achieve effective super-resolution in recorded ultrasound images.

\section{SUPER-RESOLUTION MODEL}

\subsection{Super-resolution model}

An ultrasound image is a collection of individual radio frequency (RF) signals backscattered from elementary scatters of the material. One of the most common ultrasound image display methods is the B-mode. The individual RF signal envelopes are extracted (filtered and log-compressed) and displayed in gray levels. We investigate here the theoretical framework for analysis of PSF, show how to achieve superresolution from recorded ultrasound images and provide results regarding subsequent improvement in the image. For this analysis, the following theoretical developments based on mathematical framework introduced in [13], [16] were adapted to ultrasound imaging. For easier readability, only the one dimensional case is considered; the two dimensional case is straightforward [16]. Consider two point objects, separated by a distance $d$ in the axial direction of the ultrasound beam, receiving a beam and reflecting ultrasound towards the emitting transducer. (The methodology is also valid for lateral resolution analysis). Assume the point spread function (PSF) of this imaging system is $h_{0}$, which is assumed to be causal, to have an absolute maximum, and to tend to zero at infinity. Also assume that all the parameters of the ultrasound imaging system (sound velocity, frequency, attenuation, ...) are fixed. We then deal with the distance $d$ separating the two-point sources located at $\overline{z_{0}}$ and $\overline{z_{0}}+d$ so that the echoes are $h_{0}\left(z-\overline{z_{0}}\right)$ and $h_{0}\left(z-\overline{z_{0}}-d\right)$ once received, respectively. Performing a trivial variable change, and sampling above Nyquist frequency, the received echo $y(z)$ is :

$$
y\left(z_{k}\right)=\alpha_{1} h_{0}\left(z_{k}+d / 2\right)+\alpha_{1} h_{0}\left(z_{k}-d / 2\right)+w\left(z_{k}\right)
$$

where $\alpha_{1}$ and $\alpha_{2}$ denote amplitudes of echoes and are related to the acoustic properties of the point-source; $w\left(z_{k}\right)$ is an additive zero mean gaussian white noise, with variance $\sigma^{2}$. We assume that $d$ is so small that $h_{0}\left(z_{k}-d / 2\right)$ and $h_{0}\left(z_{k}+d / 2\right)$ mainly overlap. For ultrasound imaging, we assume that $d<\lambda / 2$, where $\lambda$ is the average ultrasound wavelength. Thus if we normalize $d$ by $\lambda / 2$, we can assume that $0 \leq d<1$ (super-resolution). This implies that when $d=0$ one peak is present in combined PSF, whereas $d>0$ will exhibit two peaks. This is equivalent to classical two hypothesis testing, i.e. $H_{0}: d=0$ and $H_{0}: d>0$. Using second order Taylor expansion around $d \approx 0$, this is equivalent to

$$
\left\{\begin{aligned}
H_{0}: y\left(z_{k}\right) & =\left(\alpha_{1}+\alpha_{2}\right) \\
H_{1}: y\left(z_{k}\right) & =\left(\alpha_{1}+\alpha_{2}\right)+w\left(z_{k}\right) \\
& h_{0}\left(z_{k}\right)+\frac{\left(\alpha_{1}-\alpha_{2}\right)}{2} d \frac{\partial h_{0}}{\partial z} \\
+ & +\frac{\left(\alpha_{1}+\alpha_{2}\right)}{8} d^{2} \frac{\partial^{2} h_{0}}{\partial z^{2}}+w\left(z_{k}\right)
\end{aligned}\right.
$$

Assuming M data are available, and denoting $h_{n}\left(z_{k}\right)=$ $\left.\frac{\partial^{n} h_{0}(z)}{\partial z^{n}}\right|_{z=z_{k}}, h_{0}=\left[h_{0}\left(z_{1}\right), \ldots h_{0}\left(z_{M}\right)\right]^{T}, n=0,1,2$, and $\boldsymbol{w}=\left[w\left(z_{1}\right), \ldots, w\left(z_{M}\right)\right]^{T}$, the hypothesis tested is also :

$$
\left\{\begin{array}{l}
H_{0}: \boldsymbol{y}=\left(\alpha_{1}+\alpha_{2}\right) \boldsymbol{h}_{\mathbf{0}}+\boldsymbol{w} \\
H_{1}: \boldsymbol{y}=\left(\alpha_{1}+\alpha_{2}\right) \boldsymbol{h}_{\mathbf{0}}+\frac{\left(\alpha_{1}-\alpha_{2}\right)}{2} d \boldsymbol{h}_{\mathbf{1}}+\frac{\left(\alpha_{1}+\alpha_{2}\right)}{8} d^{2} \boldsymbol{h}_{\mathbf{2}}+\boldsymbol{w}
\end{array}\right.
$$

From this, it can be shown that the signal-to-noise ratio (SNR) is :

$$
\begin{gathered}
S N R=\frac{\left\|\left(\alpha_{1}+\alpha_{2}\right) \boldsymbol{h}_{\mathbf{0}}+\frac{\left(\alpha_{1}-\alpha_{2}\right)}{2} d \boldsymbol{h}_{\mathbf{1}}+\frac{\left(\alpha_{1}+\alpha_{2}\right)}{8} d^{2} \boldsymbol{h}_{\mathbf{2}}\right\|^{2}}{M \sigma^{2}} \\
S N R=\frac{\mu_{P F A, P D}}{M} \times \\
\frac{\left(\alpha_{1}+\alpha_{2}\right)^{2} E_{0}-\alpha_{1} \alpha_{2} d^{2} E_{1}+\left(\frac{\alpha_{1}+\alpha_{2}}{8}\right)^{2} d^{4} E_{2}}{\frac{E_{1}\left(\alpha_{1}-\alpha_{2}\right)^{2}}{4} d^{2}+\left(E_{2}-\frac{E_{1}^{2}}{E_{0}}\right) \frac{\left(\alpha_{1}+\alpha_{2}\right)^{2}}{64} d^{4}}
\end{gathered}
$$

$E_{i}=f_{s} \int_{-\infty}^{+\infty}\left|h_{i}(u)\right|^{2} d u$, where $f_{s}$ is the sampling frequency and $\mu_{P F A, P D}$ a parameter depending on the probability of false alarm $(P F A)$ and the probability of detection $(P D)$ of peaks [17]. (5) makes it possible to circumvent the knowledge of $\sigma^{2}$. In short, this shows an explicit link between $S N R$ and resolution under the Rayleigh limit. The question is now how to achieve this higher resolution.

\section{RESOLUTION ENHANCEMENT}

Here we consider a two dimensional echo signal $y$. We assume that $y$ is modeled by a second order stationary twodimensional AR process. A second order stationary twodimensional AR process (2D-AR) is defined as follows :

$y\left(n_{1}, n_{2}\right)=\sum_{\left(k_{1}, k_{2}\right) \in I} a\left(k_{1}, k_{2}\right) y\left(n_{1}-k_{1}, n_{2}-k_{2}\right)+w\left(n_{1}, n_{2}\right)$

where $I$ defines the different quarter planes; $w\left(n_{1}, n_{2}\right)$ is a complex-number gaussian field of random possibly colored noise and the parameters $a\left(k_{1}, k_{2}\right)$ are complex numbers and provide a stable system. We used complex numbers for two reasons : first the techniques developed are thus general, and second, when dealing with ultrasound, particularly for B-mode representation, it is necessary to extract envelopes of RF lines; this involves complex number signals. The parameter $a_{1}\left(k_{1}, k_{2}\right)$ and model $\left(p_{1}, p_{2}\right)$ orders may be estimated simultaneously using the relevant algorithm [18]. Once estimated, the well known power spectrum density (PSD) is given in the first quarter plane (where the parameters are denoted $\left.a_{1}\left(k_{1}, k_{2}\right)\right)$ by :

$$
P_{a r 1}\left(f_{1}, f_{2}\right)=\frac{\sigma^{2}}{\mid \sum_{k_{1}=0}^{p_{1}} \sum_{k_{2}=0}^{p_{2}} a_{1}\left(k_{1}, k_{2}\right) e^{-\left.j 2 \pi\left(f_{1} k_{1}+f_{2} k_{2}\right)\right|^{2}}},
$$


and in the fourth quarter plane (where the parameters are denoted $\left.a_{4}\left(k_{1}, k_{2}\right)\right)$ by :

$$
P_{a r 4}\left(f_{1}, f_{2}\right)=\frac{\sigma^{2}}{\mid \sum_{k_{1}=0}^{p_{1}} \sum_{k_{2}=-p_{2}}^{0} a_{4}\left(k_{1}, k_{2}\right) e^{-\left.j 2 \pi\left(f_{1} k_{1}+f_{2} k_{2}\right)\right|^{2}}} .
$$

Finally, to account for the causality and due to the equivalence between planes (see e.g. [19]), the overall PSD is given by :

$$
P_{a r 1,4}\left(f_{1}, f_{2}\right)=\frac{\sigma^{2}}{\frac{1}{2}\left[\left|A 1\left(f_{1}, f_{2}\right)\right|^{2}+\left|A 4\left(f_{1}, f_{2}\right)\right|^{2}\right]},
$$

where $\left|A 1\left(f_{1}, f_{2}\right)\right|^{2}$ and $\left|A 4\left(f_{1}, f_{2}\right)\right|^{2}$ are the denominators of eqs. (6) and (7). Thus, provided the orders (and also the parameters) are correctly estimated, the resolution of $P_{a r 1,4}$ is theoretically unlimited. These equations tell us that to improve frequency resolution it is useful to perform parametric modeling in the direct (time or space) domain (eq.(6)). This methodology can be applied to ultrasound imaging. To enhance image resolution it is useful to work on the (space domain) envelope of the image given by the RF signals. The envelope signals are provided by the magnitude of the Hilbert transform of the signals. The methodology proposed consists of performing parametric modeling on the Fourier transform (in order to be in the Fourier domain) of the Hilbert transform of the RF data. We then use eq.(8) which provides a high resolution envelope. The main advantage of this for the intrinsic resolution enhancement approach is that it applies to PSF without its explicit use or estimation. Here are the main steps of the technique.

1. Compute the Hilbert transform (of each line) of the image

2. Compute the inverse Fourier transform of a given image

3. Perform AR modeling using the above developments

4. Compute PSD to obtain well resolved envelopes.

For illustrative purposes, we consider :

$$
y\left(x_{m}, z_{n}\right)=\begin{gathered}
\alpha_{1} h_{00}\left(x_{m}+d_{x}, z_{n}+d_{z}\right) \\
+\alpha_{1} h_{00}\left(x_{m}-d_{x}, z_{n}-d_{z}\right)+w\left(x_{m}, z_{n}\right)(9)
\end{gathered}
$$

We assume that $0 \leq d_{z}<1$ and $0 \leq d_{x}<1$. $h_{00}$ is the imaging system finite support symmetric PSF and $w\left(x_{m}, z_{n}\right)$ is an additive zero mean gaussian white (or possibly colored) noise with variance $\sigma^{2}$. Let $h_{00}(z, x)=$ $\operatorname{sinc}(z) \operatorname{sinc}(x) e^{(2 \pi j c(x+y) / \lambda)}$. For readability, we only show the axial resolution. In fig.1, we show the estimated resolution (dash with 'o') using (5). For a fixed SNR, using AR modeling we estimated the optimal order and relevant achievable resolution. As can be seen, the achievable resolution was greater than that provided by (5). The following figures show the results using a thread embedded in a gel and some in vivo

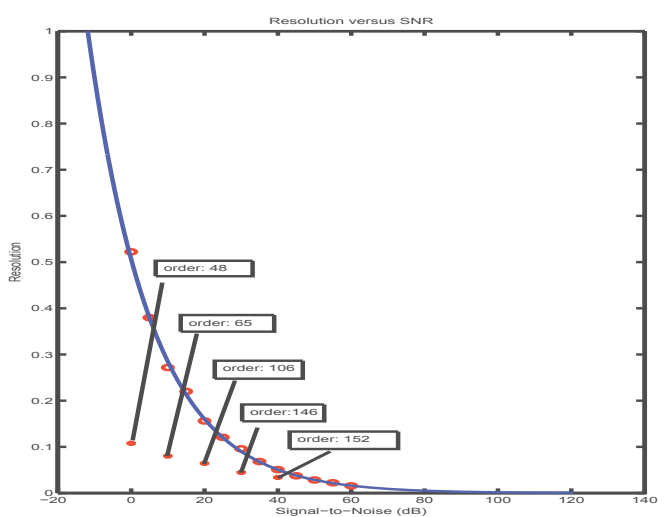

Fig. 1.

For a fixed SNR, AR modeling was used to estimate the optimal order and relevant achievable resolution. As can be seen, the achievable resolution was greater than that provided by (5).

materials. The images shown in fig. 2 are transverse images of a single thread embedded in a gel. The arbitrary expected theoretical image is shown at the top; the lower left image is the original $20 \mathrm{MHz}$ ultrasound image, and the lower right image shows the result after using the proposed method. As can be seen, the processed image is closer to the expected theoretical image. The images shown in fig. 3 compare 20
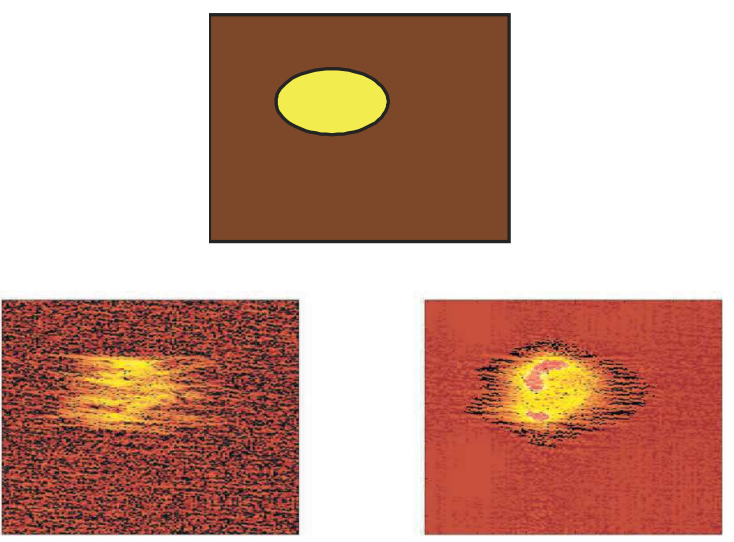

Fig. 2.

Comparison between expected theoretical image (top), original $20 \mathrm{MHz}$ ultrasound image (lower left) and processed image (lower right) obtained from the original ultrasound image.

$\mathrm{MHz}$ ultrasound images of a mouse kidney. The original ultrasound image is shown on the left, and the right image 
shows the result after using the proposed method. As can be seen, more detail is provided by the processed image.
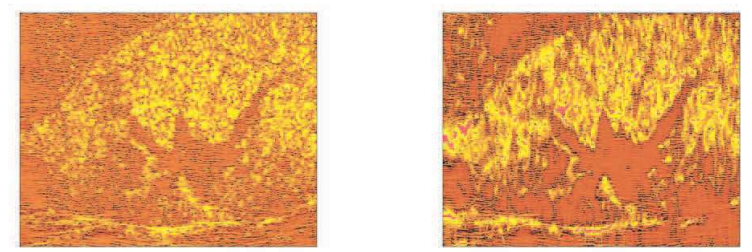

Fig. 3.

Comparison between original $20 \mathrm{MHz}$ ultrasound image of a mouse kidney (left) and processed image (right) obtained from the original ultrasound image.

\section{CONCLUSION AND GENERAL REMARKS}

After a brief overview of SR in medical imaging, we have summarized super-resolution theory and have shown how to enhance resolution from recorded ultrasound images and provided results regarding subsequent improvement in the image. This algorithm was based on correct estimation of model order and parameters. The results demonstrate that such parametric modeling, combined with the proposed ultrasound imaging technique, is an interesting way to enhance ultrasound image resolution. Further intensive investigations are in progress to evaluate the theoretical and quantitative performance of this technique.

\section{REFERENCES}

[1] S. Baker and T. Kanade, "Limits on super-resolution and how to break them," IEEE Trans. Patt. Anal. and Mach Rec, vol. 24, pp. 1157-1183, 2002.

[2] S.C. Park, S.C. Park, and M.G. Kang, "Super-resolution image reconstruction : A technical overview," IEEE Signal Proces. Mag., pp. 21-36, May 2003.

[3] R. Hardy, "A fast image super-resolution algorithm using an adptive wiener filter," IEEE Trans. Image Proces., vol. 16, pp. 2953-2964, 2007.

[4] R. Laforest, D.J. Rowland, and M.J. Welch, "Micropet imaging with nonconventional isotopes," IEEE Trans. Nuclear Science, vol. 49, pp. 2119-2126, 2002.

[5] Special issue, "Special issue on novel equipment for ultrasound research," IEEE Trans. Ultras. Ferroel. Frequency Cont, vol. 53.

[6] S. Maramraju, S. H.and Junnarkar, B. Ravindranath, S. Southekal, and S. D.and Lenz W.and et.al Stoll,
S.and Smith, "An mr compatible pet scanner based on ratcap for small animal imaging at 9.4 t," in Proc. of IEEE Nuclear Science Symposium, 2008.

[7] L.R. Wilson, D.W.and Furenlid, H.H. Barrett, and Y.C. Chen, "A new pet system for small-animal imaging," in Proc. of IEEE Nuclear Science Symposium, 2004.

[8] Foster F. S., Pavlin, C. J., and Harasiewicz K.A., “Advances in ultrasound biomicroscopy,", Ultras. in Med. Biol., vol. 26, pp. 1-27, 2000.

[9] G.T. Clement, J. Huttunen, and K. Hynynen, "Superresolution ultrasound imaging using back-projected reconstruction," J. Acoust. Soc. Am., vol. 118, pp. 3953-3960, 2006.

[10] P. Blomgren and G. Papanicolaou, "Super-resolution in time reversal acoustics," J. Acoust. Soc. Am., vol. 111, pp. 230-248, 2002.

[11] R. Jirik and T. Taxt, "High-resolution ultrasonic imaging using two-dimensional homomorphic filtering,", IEEE Trans. Ultras. Ferroel. Frequency Cont, vol. 53, pp. 1440-1448, 2006.

[12] L. Rayleigh, "On the manufacture and theory of diffraction-grattings," Philos. Mag., vol. 47, pp. 193205, 1874.

[13] A. Van den Bos, "Ultimate resolution : A mathematical framework," Ultramicroscopy, vol. 47, pp. 298-306, 1992.

[14] E. Bettens, D.V. Dyck, A.J. den Dekker, J. Sijbers, and A. Van den Bos, "Model-based two-object resolution from observation having couting statistics," Ultramicroscopy, vol. 77, pp. 37-48, 1999.

[15] A.J. den Dekker, "Model-based optical resolution," IEEE Trans. Instr. Meas, vol. 46, pp. 798-802, 1997.

[16] M. Shahram and P. Mylanfar, "Statistical and information-theoric analysis of resolution in imaging," IEEE Trans. Inf. Theory, vol. 52, pp. 3411-3436, 2006.

[17] M. Shahram and P. Mylanfar, "Imaging below the diffraction limit: A statistical analysis," IEEE Trans. Image Proc, vol. 13, pp. 677-689, 2004.

[18] D. Kouamé, C. Garnier, J.M. Grégoire, and J.M. Girault, "Multidimensional complex number parametric model order and parameters estimation," in Proc. of IEEE ICASSP, 2006.

[19] Steven M. Kay, Modern Spectral Estimations, PrenticeHall signal Processing Series, 1988. 